\title{
VARIANTES Y EQUIVALENTES FUNCIONALES DE AL FINAL: LOS REFORMULADORES DE RECAPITULACIÓN EN EL HABLA SANTIAGUINA ${ }^{1}$
}

\author{
VARIANTS AND FUNCTIONAL EQUIVALENTS OF AL FINAL: THE \\ REFORMULATORS OF RECAPITULATION IN THE SANTIAGO, \\ CHILE SPEECH
}

\author{
ABELARDO SAN MARTÍN NÚÑEZ \\ Sociolingüística y pragmática \\ Universidad de Chile \\ asmartin@uchile.cl
}

\section{RESUMEN}

En este trabajo se analiza el empleo de los reformuladores de recapitulación, en una muestra del español hablado en Santiago de Chile, desde el punto de vista sociolingüístico. En particular, se describe el comportamiento de los marcadores del discurso, que cumplen la mencionada función, en una muestra estratificada de 120 entrevistas de hablantes santiaguinos y se establece la distribución sociolingüística de su empleo. Asimismo, se desarrolló la conceptualización teórica de los marcadores del discurso de Cortés (1998), Martín Zorraquino y Portolés (1999), Portolés (2001), y Carbonero y Santana (2010). En el análisis se consideró la función "reformulación de recapitulación mediante marcadores" como un caso de variable sociolingüística con diferentes valores o variantes (recapituladores). De modo consecuente, su uso se correlacionó con los factores sociodemográficos (sexo, edad y grupo socioeconómico) de los sujetos entrevistados, con base en su conmutabilidad funcional a nivel discursivo. Los resultados muestran que los recapituladores constituyen un paradigma amplio de marcadores, aunque con un claro predominio de al final y o sea, que son los más frecuentes; asimismo, o sea como marcador recapitulativo es más usado por los informantes del grupo socioeconómico medio alto.

Palabras clave: marcadores del discurso, reformulación, recapitulación, variación discursiva, sociolingüística, español de Chile.

\footnotetext{
${ }^{1}$ La realización de este artículo contó con el apoyo del Concurso U-Apoya-Ayuda de Viaje de la Vicerrectoría de Investigación y Desarrollo de la Universidad de Chile. En este trabajo se sintetizan los resultados más relevantes de una de las secciones de nuestra tesis doctoral inédita, "Variación sintáctica y discursiva en el español hablado en Santiago de Chile. Análisis sociolingüístico del queísmo, el dequeísmo, el discurso referido y los marcadores de reformulación”, dirigida por el Dr. Humberto López Morales.
} 


\begin{abstract}
In this paper the use of the reformulators of recapitulation in a sample of Spanish spoken in Santiago, Chile, from a sociolinguistic point of view is analyzed. Particularly, the behavior of discourse markers that meet this function is described and at the same time the sociolinguistics distribution of this use is established, in a stratified sample of 120 interviews. Also, the theoretical conceptualization of discourse markers of Cortés (1998), Martín Zorraquino \& Portolés (1999), Portolés (2001) and Carbonero \& Santana (2010) was developed. In the analysis, was considered the function "reformulation of recapitulation with markers" as a case of sociolinguistic variable with different values or variants (recap's markers). Consequently, his use were correlated with the sociodemographic factors (sex, age and socioeconomic group) of subjects interviewed, based on their functional commutability in discursive level. The results show that recap's markers constitute a wide paradigm, but with a clear predominance of al final and $o$ sea, they are the most frequent; also, $o$ sea as recapitulative marker is more used by informants of upper middle socioeconomic group.
\end{abstract}

Keywords: Discourse markers, reformulation, recapitulation, discursive variation, sociolinguistics, Chilean Spanish.

Recibido: 13.05.2015. Aceptado: 30.09.2015.

\title{
1. INTRODUCCIÓN
}

A pesar de la importancia del papel que cumplen los marcadores del discurso - en la comprensión de los enunciados (cf., entre otros, Schiffrin, 1987 y 2003; Anscombre y Ducrot, 1994; Martín Zorraquino y Montolío, 1998; Martín Zorraquino y Portolés, 1999; Portolés, 2001; y Loureda y Acín, 2010), en español, la variación social y dialectal de su empleo constituye una de las líneas de indagación menos exploradas (Carbonero y Santana, 2010). El objetivo general de este trabajo - de naturaleza cuantitativa y correlacional- es analizar el comportamiento pragmático-discursivo y la estratificación sociolingüística de los reformuladores de recapitulación, en una muestra de 120 entrevistas realizadas a informantes santiaguinos. De manera consecuente, los objetivos específicos del estudio son: a) identificar los marcadores que cumplen la función de reformulación de recapitulación en la muestra; b) determinar su frecuencia de empleo y c) correlacionar dichas frecuencias con las variables sociodemográficas: sexo, edad y grupo socioeconómico de los informantes. Asimismo, las hipótesis sometidas a contrastación empírica en este trabajo son: a) al final es el reformulador de recapitulación que se emplea, preferentemente, en el habla de la comunidad bajo estudio, aunque existe un paradigma amplio de marcadores recapitulativos y b) los factores sociodemográficos 
de los sujetos inciden de manera irregular en el empleo de los reformuladores de recapitulación relevados en la muestra.

\section{MARCO CONCEPTUAL}

\subsection{Los marcadores del discurso desde el punto de vista sociolingüístico}

Este trabajo se enmarca en las directrices teóricas y metodológicas generales de la sociolingüística variacionista desarrollada por Labov (1983), con las necesarias adaptaciones al estudio de la variación en el ámbito discursivo (Macaulay, 2003). Los estudios en el ámbito del variacionismo han demostrado, en general, la influencia de factores sociales como el sexo, la edad, el nivel educacional o el grupo socioeconómico de los hablantes, en conjunto con la de factores internos, en el comportamiento lingüístico (Bright, 1998; Silva-Corvalán, 2001; López Morales, 2004; Blas Arroyo, 2005; Moreno Fernández, 2009 y Serrano, 2011). Silva-Corvalán, en este sentido, señala que "la variación lingüística no es aleatoria sino que está condicionada tanto por factores internos al sistema de la lengua como por factores sociales externos a ella" (2001: 2). Según este principio, la variación sociolingüística ha sido definida como "la alternancia de dos o más expresiones de un mismo elemento, cuando ésta no supone ningún tipo de alteración o cambio de naturaleza semántica y cuando se ve condicionada por factores lingüísticos y sociales" (Moreno Fernández, 2009: 39). No obstante, la extensión del concepto de variable sociolingüística al estudio de fenómenos más allá del nivel fonético planteó una serie de dificultades, especialmente, a partir del trabajo de Lavandera (1978) donde se cuestionó explícitamente dicha extensión, arguyendo que no estaba garantizado en el estudio de las mencionadas variables el principio de invariabilidad a nivel semántico. A pesar de las diferentes posturas en torno a este debate, en principio, la variación lingüística "definida como el uso alterno de formas diferentes de decir lo mismo, se puede encontrar prácticamente en todos los niveles de la lengua, desde el más concreto (fonético-fonológico) al más amplio (discurso, por ejemplo), pasando por la gramática y el léxico" (Moreno Fernández, 2009: 22).

Como en trabajos anteriores (San Martín 2004-2005, 2011 y 2013 y Rojas, Rubio, San Martín y Guerrero, 2012), aquí se sugiere la posibilidad de aplicar el concepto de "variable lingüística" -en un sentido amplio- al estudio de los marcadores del discurso. En relación con el problema de que se ocupa el presente artículo, Cortés (1998: 160) concluye que el estudio variacionista de los marcadores es posible, si se cumple el requisito de contrastar las frecuencias de conectores que cumplan las mismas funciones, lo que trae aparejadas no pocas dificultades. Para el análisis sociolingüístico de los marcadores, es preciso que se asocien las características de los informantes con aquellas partículas que desempeñen una función 
común en el discurso (Cortés, 1998: 154). De acuerdo con la perspectiva del autor del presente trabajo, una vez identificadas las partículas que cumplan una función pragmática equivalente, la aplicación del concepto de variable sociolingüística al estudio de los marcadores del discurso es factible, "puesto que este tipo de formas parece cumplir con las condiciones propuestas en el marco del variacionismo, es decir, consistir en 'formas alternativas de decir lo mismo" (San Martín 2004-2005: 213). Por su parte, Carbonero y Santana (2010: 516-517) señalan la carencia de trabajos que aborden la variación espacial y social de los marcadores del discurso, aunque, al mismo tiempo, destacan el interés de esta línea de trabajo para el mejor conocimiento de estas unidades de procesamiento.

\subsection{Los reformuladores de recapitulación en la lengua española}

Los marcadores del discurso son unidades lingüísticas de enlace de carácter invariable, que no ejercen una función sintáctica a nivel oracional y cuya función primordial es orientar las inferencias que se realizan en la comunicación (Portolés, 2001: 25-26) ${ }^{2}$. Para el presente estudio, se adoptará, principalmente, la perspectiva teórica sobre los marcadores del discurso de Martín Zorraquino y Portolés (1999) y Portolés (2001). Desde dicha perspectiva, los marcadores de reformulación "presentan el miembro del discurso que introducen como una nueva formulación de un miembro anterior” (Martín Zorraquino y Portolés, 1999: 4121). La clasificación de los reformuladores propuesta por los mencionados autores incluye los siguientes subtipos: explicativos, rectificativos, de distanciamiento y recapitulativos. Según su función específica, los reformuladores de recapitulación muestran al miembro del discurso en el cual se insertan como una conclusión o recapitulación a partir de un miembro anterior o una serie de ellos, conservando la misma orientación argumentativa de los miembros anteriores - por ejemplo, en suma, en conclusión, en resumen y en sintesis- o incorporando miembros con una orientación opuesta -por ejemplo, en resumidas cuentas, en definitiva, en fin, total, vamos, al fin y al cabo y después de todo (Martín Zorraquino y Portolés, 1999: 4122).

Por su parte, Garcés (2003 y 2011) destaca que la reformulación es un "procedimiento de organización del discurso que permite al hablante volver sobre un segmento anterior para reinterpretarlo y presentarlo de manera distinta" (2011: 90), que responde a cinco motivaciones de los hablantes: explicar, recapitular,

\footnotetext{
${ }^{2}$ Martín Zorraquino (1998: 35-51) plantea que, pese a la heterogeneidad de los marcadores del discurso, relativa tanto a las categorías a las que pertenecen como a su distribución en el discurso y a sus funciones pragmáticas, dichas unidades presentan, entre otras, las siguientes propiedades comunes: a) no pueden ser sustituidas por elementos pronominales o deícticos, b) no pueden ser sometidos a interrogación parcial, c) no admiten la coordinación con elementos equifuncionales y d) no pueden ser reemplazados por elementos pronominales que reproduzcan la totalidad, o la parcialidad, de la oración en la que se sitúan.
} 
reconsiderar, distanciarse o corregir (2003: 114). Aplicando los supuestos de la lingüística textual de Gülich y Kotschi (1983 y 1995) y Rossari (1994), además, Garcés (2003 y 2011) distingue entre la reformulación parafrástica, en la que se plantea una equiparación semántica y pragmática entre los segmentos reformulado y reformulador, y la no parafrástica, en la que se propone un distanciamiento de diferente grado (mínimo, medio y máximo) entre ambos segmentos. Así, para esta autora, la reformulación es un "proceso de reinterpretación de un segmento previo, explícito o implícito, que, según la relación entre los miembros y el tipo de marcador empleado, puede ir desde la equivalencia hasta el distanciamiento" (Garcés, 2008: 82) ${ }^{3}$. Su clasificación considera cinco tipos de marcadores de reformulación, de acuerdo con las motivaciones señaladas más arriba: explicación, rectificación, recapitulación, reconsideración y separación (Garcés, 2008: 86). Por lo que se refiere al tipo de reformulación que nos ocupa, en la recapitulación, el miembro reformulador introduce una síntesis del contenido previo (mediante marcadores como en suma, en sintesis y en resumen).

Por lo que refiere al español de Chile, en Rojas et al. (2012: 115-116) se señala el siguiente inventario de marcadores recapitulativos, en un corpus de 72 entrevistas de Santiago: al final, en definitiva, al fin y al cabo, o sea, a las finales, total, a la final, en fin y al final de cuentas. Por otro lado, en su relevamiento de los marcadores del discurso empleados en 12 entrevistas de la norma culta santiaguina, Valencia (2014b) señala que los reformuladores constituyen el tipo de marcador menos utilizado, entre los que halló ejemplos de los recapitulativos en fin, en definitiva y total ${ }^{4}$.

\section{METODOLOGÍA}

\subsection{Corpus}

El corpus que servirá de base para el presente estudio corresponde a 120 entrevistas sociolingüísticas pertenecientes al corpus del grupo de Estudio Sociolingüístico del Español de Chile (ESECH)5. Las entrevistas fueron realizadas a hablantes

\footnotetext{
${ }^{3}$ Según Garcés (2008: 67), la capacidad de replantear enunciados previos mediante otros que nos parecen más expresivos o adecuados para nuestra propia intención es una propiedad de la competencia metapragmática de los hablantes. Este aspecto también ha sido destacado por Portolés (2004).

${ }^{4}$ No obstante, es importante precisar que la misma autora declara que, debido a motivaciones contrastivas, en su estudio no se relevaron usos idiosincrásicos o dialectales (Valencia, 2014a: 7-8). Por lo tanto, su análisis solo se limitó a identificar el empleo del inventario de marcadores informado en Martín Zorraquino y Portolés (1999). Esto plantea una diferencia de estrategia de indagación fundamental con respecto a la adoptada en el presente trabajo.

${ }^{5}$ El ESECH es un grupo de investigación del Departamento de Lingüística de la Facultad de Filosofía y Humanidades de la Universidad de Chile coordinado por nosotros.
} 
santiaguinos, entre 2005 y 2011, por los estudiantes en la asignatura de Sociolingüística de los programas de Licenciatura en Lengua y Literatura Hispánicas y Licenciatura en Lengua y Literatura Inglesas de la Universidad de Chile. En la situación de entrevista, los entrevistadores debían tratar de superar la "paradoja del observador" consiguiendo, de esta forma, una muestra significativa de discurso natural grabado (estilo vernáculo) de hablantes representativos de la comunidad de habla en estudio (Labov, 1983). El número de entrevistas antes indicado totaliza, aproximadamente, 116 horas de grabación, cuya transcripción fue exhaustivamente revisada, sin exclusión de ninguna de las secciones del instrumento.

\subsection{Población y muestra}

En la investigación se consideró la población constituida por hombres y mujeres de la Región Metropolitana de más de 20 años de edad ${ }^{6}$. El cuestionario se aplicó a una muestra del tipo "muestra por cuotas con afijación uniforme", en la que se divide a la población en estratos o categorías y se asigna una cuota a cada uno de los distintos estratos (López Morales, 1994: 58). Por lo que respecta al tamaño de la muestra, se optó por conformarla con un número de informantes considerado, en general, como suficiente en la bibliografía sociolingüística (cf. Trudgill y Hernández Campoy, 2007, s. v. representatividad y López Morales, 1994: 52). La muestra, así conformada, comprende un total de 120 entrevistas realizadas a igual número de sujetos, distribuidos como se indica en la Tabla I que se muestra a continuación:

Tabla I. Proporción de sujetos según características sociodemográficas de sexo, edad y grupo socioeconómico.

\begin{tabular}{|c|c|c|c|c|c|c|c|}
\hline & \multicolumn{2}{|c|}{$20-34$} & \multicolumn{2}{|c|}{$35-54$} & \multicolumn{2}{|c|}{55 y más } & \multirow[b]{2}{*}{ Total } \\
\hline & $\mathrm{H}$ & M & $\mathrm{H}$ & M & $\mathrm{H}$ & M & \\
\hline Medio alto & 5 & 5 & 5 & 5 & 5 & 5 & $=30$ \\
\hline Medio & 5 & 5 & 5 & 5 & 5 & 5 & $=30$ \\
\hline Medio bajo & 5 & 5 & 5 & 5 & 5 & 5 & $=30$ \\
\hline Bajo & 5 & 5 & 5 & 5 & 5 & 5 & $=30$ \\
\hline & 20 & 20 & 20 & 20 & 20 & 20 & $=120$ \\
\hline
\end{tabular}

\footnotetext{
${ }^{6}$ En relación a los criterios de asignación de estatus de hablante nativo de Santiago de Chile, hemos adaptado la propuesta de Prieto (1995-1996), por lo que se aplicaron las siguientes restricciones en la selección de los sujetos: 1) haber nacido y residido en forma ininterrumpida en Santiago, 2) haber nacido y residido en Santiago la mayor parte de sus vidas, salvo por periodos que sumados no superen los cinco años y 3) haber residido en forma ininterrumpida en Santiago desde los cinco años de edad.
} 


\subsection{Procedimiento de estratificación social utilizado en ESECH}

Para la estratificación de los sujetos que conforman la muestra del estudio, se utilizó el sistema de adscripción de estatus social empleado en ESECH que se basa en la asignación del puntaje -que se indica entre paréntesis- a los informantes, de acuerdo con las siguientes variables: a) nivel educacional (3), b) profesión u ocupación (2) y c) comuna de residencia (1). La explicación en detalle de dichas variables se encuentra en Prieto (1995-1996), cuya propuesta fue perfeccionada y actualizada con base en los estudios acerca de la realidad socioeconómica chilena de la empresa de Investigaciones de Mercado y Opinión Pública (ADIMARK, 2003), la Descripción Básica de los Niveles Sociales realizada por el Instituto Consultor en Comercialización y Mercado (ICCOM, 2005) y la propuesta de estratificación de la Asociación de Investigadores de Mercado (AIM, 2008) 7 . A partir de la asignación de los mencionados puntajes, se distinguieron cuatro grupos socioeconómicos, definidos según los rangos siguientes: a) Medio alto (MA): 42 - 36, b) Medio (M): $35-27$, c) Medio bajo (MB): $26-18$ y d) Bajo (B): $17-6^{8}$. El intervalo de cada rango responde a la mayor coincidencia con los puntajes que han establecido los estudios sociológicos y de mercado para la clasificación de estratos socioeconómicos, aunque con las debidas adaptaciones, puesto que en esta muestra no se incluyen ni el grupo de la extrema pobreza ni el de la extrema riqueza (cf. San Martín y Guerrero, en prensa).

\subsection{Procedimiento analítico}

Nuestro análisis de la función discursiva "reformulación de recapitulación mediante marcadores" se concentró en la determinación de las frecuencias de ocurrencia de cada una de las partículas específicas, que cumplen dicha función en las entrevistas de la muestra. Asimismo, de modo coherente con los objetivos planteados, se observará la incidencia de los siguientes factores sociodemográficos de los hablantes: sexo, edad y grupo socioeconómico. Por lo que se refiere a la descripción de los procedimientos estadísticos aplicados, en este trabajo se siguen las

\footnotetext{
${ }^{7}$ Cabe precisar que el modelo de nivel socioeconómico fue adaptado de estos estudios de mercado en función de los objetivos que se propone el grupo ESECH, que busca definir un perfil más bien sociocultural de la comunidad lingüística en estudio. En consideración a esto, para la estratificación socioeconómica, una de las variables más determinantes fue el nivel educacional y, por esta razón, es la que asigna el mayor puntaje. Asimismo, el nivel de ingresos demuestra muy poca relevancia para los estudios de mercado antes mencionados, por ello no fue considerado en la determinación de los perfiles socioeconómicos.

${ }^{8}$ En la selección de las entrevistas del corpus se cauteló la consistencia entre las variables antes mencionadas de manera que respondieran a lo que Prieto (1995-1996), siguiendo a Lenski (1954), denominara como congruencia de estatus.
} 
sugerencias e indicaciones de Moreno Fernández (1990), López Morales (1994) y Hernández Campoy y Almeida (2005). El análisis estadístico atenderá a dos niveles de observación: a) en términos descriptivos, según las frecuencias absolutas y los porcentajes de frecuencia de cada recapitulador y b) en términos interpretativos o inferenciales, con base en la comparación entre las medias o tendencias centrales de los marcadores más frecuentes, esto es, de aquellos con más de 25 ocurrencias. El paquete estadístico al que hemos recurrido para la estadística inferencial es el SPSS (Statistical Package for the Social Sciences), versión 15.0 para Windows, específicamente, la prueba Análisis de varianza ANOVA. En atención a que la distribución de los datos, en algunos casos, pudiera ser anormal, se complementará dicho análisis con su análogo de tipo no paramétrico, esto es, Anova de Kruskal Wallis. En ambos casos, el grado de significación se definirá en el 5\%, según el cual $\mathrm{p}=<0,05$ será estadísticamente significativo.

\section{PRESENTACIÓN Y ANÁLISIS DE LOS RESULTADOS}

\subsection{Análisis discursivo de los reformuladores de recapitulación relevados en el corpus}

Los recapitulativos, como ya se indicó, introducen una síntesis a partir de un segmento o de una serie de segmentos discursivos previos. Dicho segmento recapitulativo puede estar coorientado o antiorientado, argumentativamente, respecto de los segmentos sintetizados, aunque el significado procedimental básico inferible de este tipo de reformuladores es que el enunciado siguiente es el resultado final del contenido precedente. En la muestra analizada se recogieron 412 casos de empleo de reformuladores recapitulativos que se reparten en el siguiente paradigma con las frecuencias absolutas que se señalan: al final (184), a la final (3), a las finales (15), a fin de cuentas (1), al final de cuentas (2), al fin y al cabo (1), al final y al cabo (6), al final al cabo (6), a la final y al cabo (1), o sea (134), total (19), en fin (20), en definitiva (17), en resumen (1) y cuento corto (2). En el Gráfico 1 se presentan las frecuencias porcentuales de estos marcadores en los materiales analizados: 


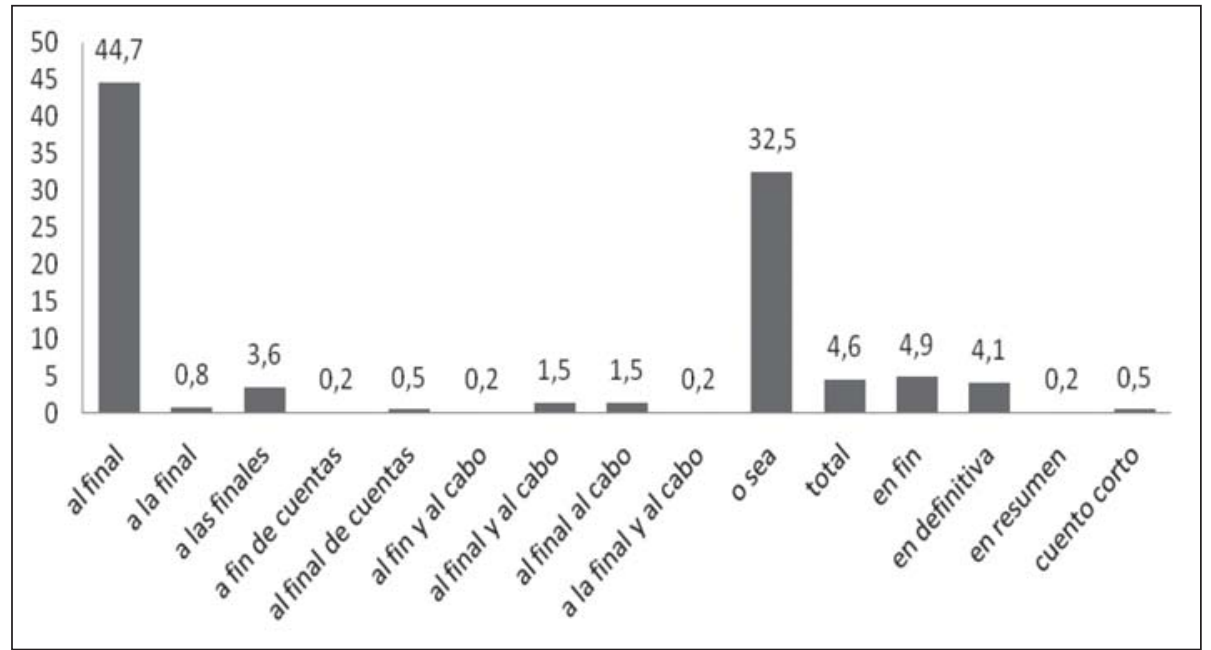

Gráfico 1. Porcentaje de frecuencia de los marcadores recapitulativos en el corpus.

Los ejemplos que se presentan, a continuación, ilustran el empleo de los marcadores de recapitulación en el corpus's:

\section{Al final}

1) por ahí tengo/ como la/ esa/ ese/ el deseo// que creo que todo el mundo debe tener pero// pero no vivo mi vida en base a eso/ o sea no ando jugando esas/

${ }^{9}$ Respecto de la transcripción de los ejemplos, es necesario señalar las siguientes convenciones gráficas: 1) cuando corresponda a ejemplos de mayor extensión, E = entrevistador e I = informante; 2) como se trata de variables no fonéticas y, por lo tanto, la pronunciación no es el foco del análisis, el texto correspondiente a cada ejemplo se transcribe en ortografía convencional, incluidos los acentos gráficos; 3) con el propósito de no desnaturalizar demasiado la representación del habla chilena, se ha conservado la manifestación gráfica de determinados usos característicos del español hablado en Chile, como el uso del voseo paradigmático con elisión de -s, por ejemplo, estái, soi, viví (estáis, sois, vivís con valor de segunda persona del singular, tú) y el empleo de marcadores del discurso, como ¿cachái? (“¿entiendes?”) y po (pues); 4) para la mejor comprensión de los ejemplos incluidos en este estudio, se ha suprimido el sistema de etiquetas pertenecientes al Standard Generalized Markup Language (SGML) contenidas en el Text Encoding Initiative (TEI), que se emplea en la transcripción de las entrevistas de ESECH, excepto las pausas que se señalan con /. Al final de cada ejemplo se indica, entre paréntesis, el código del informante, según las siguientes convenciones: grupo socioeconómico ( $\mathrm{MA}=$ medio alto, $\mathrm{M}=$ medio, $\mathrm{MB}=$ medio bajo, $\mathrm{B}=$ bajo), grupo etario (III = adultos mayores de 55 años y más, II = sujetos adultos de edad intermedia entre 35 y 54 años y I = hablantes jóvenes de entre 20 y 34 años $)$ y sexo $(\mathrm{M}=$ mujer y $\mathrm{H}=$ hombre $)$. A continuación del sexo se señala el número correlativo del sujeto en el corpus. 
encuentro absurdo andar gastando plata todas las semanas para jugar eso si al final la expectativa de que uno se lo gane son mínimas (MAIIM171),

2) mi marido es de gente de campo/ todas sus hermanas entonces son buenas/ para cocinar/ para hacer el pan amasado/ entonces de repente cuando íbamos a la casa de de mi marido que tienen ahí en el campo que es una casa muy humilde/ pero es grandota y llega mucha gente todos sus hermanos/ ee/ llegamos allá/ hacemos mermelada ee que el pastel de choclo que el pan amasado/ al final vamos a puro trabajar po/ porque no falta que las humitas/ el pastel de choclo (MIIIM137),

3) lo mejor que me ha pasado fue cuando nació mi hijo/ porque no/ esa sensación de sentir que eres mamál a pesar de todo lo que había pasado/ no sél eso fue lo mejor que me ha pasado/ aparte que yo programé// cuando yo supe que estaba embarazada programé el parto/ quería tener a mi hijo sin anestesia/ fue un trabajo súper complicado pero/ pero al final la recompensa que tienes es demasiado hermosa (MIM108),

3) así que olvídate tuve que// consultamos inmediatamente la primera hora en el otro la oportunidad que pudimos// ee/ o sea inmediatamente porque fue un día viernes una cosa así// el día lunes lo llevamos a un dentista// llevamos las radiografías y todo bueno po// al finall gracias a Dios no era nada malo porque lo que pasa es que tuvo un desarrollo/ de dientes mayor al que tiene habitualmente las personas (MBIIM076).

\section{A la final}

5) la verdad es que// estái tan poco tiempo ahí/ que a la final te da lo mismo porque/ tu casa a la final es el dormitorio/ tú llegas a tu casa a dormir/ y después sales temprano en la mañana a trabajar (MBIIH065).

\section{$A$ las finales}

6) porque/ igual/ no sé po/ igual como una lata yo creo para ellos porque/ andar con los papeleos que para allá/ el gasto de plata/ y/ toda esa onda/ y aparte es un papel que te compromete no más/ sí a las finales/ son tus firmas y nada más $\mathrm{po} /$ no te compromete a nada/ que vai a tener una vida feliz// que vai a ser/ el mejor papá/ la mejor mamál ¿̨cachái? (MBIH049),

7) y la rutina me cagó a mí/ la rutina fue la que me cagó/ el trabajo/ que trabajábamos los dos aquí en la casa y/y nada po/ y puras peleas/ que peleaba por la 
Variantes y equivalentes funcionales de al final: los reformuladores de recapitulación en el habla santiaguina / A. SAN MARTín

plata/ que quién va a comprar/ que quién hace esto esto otro/ a las finales/ era yo el hombre de la casa po/ y toda esa huevada me aburrió/ me aburrió/ y por eso/ cuando conocí a esta persona/ no no me arrepentí para nada (BIIM028),

8) ellos estuvieron en la casa con la orquesta/ entonces fue como ";ay la media fiesta!"/ si lo único que nos embarró la onda que ese día llovió// y quedó la escoba pero a las finales igual lo pasamos bien (BIIM025).

\section{A fin de cuentas}

9) EM que es como la cara clásica del dominio de la derecha económica ee chilena/ LT que también es un diario de corte liberal también pro empresarial y el resto de la prensa en un// de que no existe/ o sea o es la prensa del gobierno que es LN que trata de ser crítico pero a fin de cuentas dice lo que el gobierno quiere/ TC que también a estas alturas del partido lo único que hace es prácticamente se centró como un medio de comunicación netamente ee mm casi de corte oficialista (MAIH145).

\section{Al final de cuentas}

10) generalmente te va a contratar una empresa como fabricante de como co una una empre/ una fábrica de muebles te va a contratar/ entonces te van a pagar en la medida que vendas/ entonces al final de cuentas/ esas son las expectativas las expectativas siempre/ mías fueron que el sueldo me lo tenía que hacer yo (MAIIIM185).

\section{Al fin y al cabo}

11) yo creo que no debería existir el aborto/ porque ya si/ puta/ no querí no quieres o no puedes en muchos casos o en el caso soy soy/ bueno siendo mujer ultrajada y de eso nace un hijo/ al fin y al cabo es una vida y si ya no la quieres tener por último dala en adopción (BIH002).

\section{Al final y al cabo}

12) las campañas electorales son realmente una estupidez absurda que pueden inventar los políticos/ definitivamente una estupidez porque en vez de ver solución a los problemas/ hacen más conflicto para tratar de alzar más su puesto y al final y al cabo nunca arreglan progresivamente la vida que estamos llevando po (BIH002). 


\section{Al final al cabo}

13) se puede decir que me gustaba la ideología y me dejé guiar su cierto tiempo por ellos/ pero al final al cabo me di cuenta que no era lo mío ¿cachái? (BIH002).

\section{A la final y al cabo}

14) las dos sí a la final y al cabo son fiestas/ las tení que pasar bien nomás/ es que hay que reírse sacar las penas ya que ya se va el año empezái año nuevo/ entonces hay que pa/ pasarla bien po en familia (BIH002).

\section{O sea}

15) pero pero eso es la parte académica ahora en la parte en la parte ee ee llamémosle así familiar yo creo que fue una gran alegría por ejemplo el día que me casé// ya/ ee después el día que tuve nues/ tuvimos nuestro primer hijo/ el día que tuvimos el segundo hijo ee/ después cuando hicieron la primera comunión $o$ sea todos esos hitos ¿̨no cierto? (MAIIIH179),

16) he pasado por periodos/ pero los últimos que me ha dado/ me ha dado obsesión en los últimos diez años/ no sé/ la Simone de Beauvoir/ Paul Auster// después el Ian McEwan/ la Virginia Woolf que estuve leyendo una biografía de ella pero bueno/ buena/ y leí// hace poco que me lo había leído La Montaña Mágica de Mann que me encantó no lo había leído que me encantó/ y ahora estoy con Ulises como por décima vez que lo empiezo pero esta vez lo voy a terminar/ ya caché que lo voy a terminar/ pero eso/ o seal/ leo de todo contemporáneos y un poco más viejos (MAIIM169),

17) no sentí campanitas ni dije “¡oh!”/ no/ pero fue lindo o sea todo/ porque me acuerdo del momento que me fui a la clínica/ de las personas que estaban/ de mi cesárea/ cuándo volví de la cesárea/ cuándo me la tra/ todo// todo/ ¿me entiendes? o sea es un recuerdo precioso (MIIIM141).

\section{Total}

18) en eso consiste la soriasis nada más// y el tratamiento que tuve era fue regular la velocidad del crecimiento de la evolución de las células pero por lo menos/ estuve unos tres meses súper bien y ahora estoy bajo control nomás po// bajo control/ y bien también/ o sea/ total ee las enfermedades son parte del ser hu- 
Variantes y equivalentes funcionales de al final: los reformuladores de recapitulación en el habla santiaguina / A. SAN MARTíN

mano/ y hay que aprender a quererse hay que aprender a aceptarlas y vivir con ellas/ yo convivo con mi enfermedad (MAIIIH180),

19) me da veinte mil pesos al mes/ a veces me da diez mil pesos y ¿qué voy a hacer con eso para comer para mantenerme para comprar mis remedios que paso enferma?/ entonces no me alcanza ni para los remedios/ los veinte mil pesos me duran unos dos tres días y “¡chao!" y "te fuiste”/ y yo tengo que andar pidiendo/ no sí en serio yo salgo a pedir/ no tengo vergüenza para nada/ voy al centro voy a los restoranes adonde sea por ahí pido/ total no me da vergüenza porque para pedir no se tiene vergüenza porque uno tiene que comer po (BIIIM043).

\section{En fin}

20) y por ahí entonces se ha producido/ ee de que no han entregado todo lo que tenían que entregar/ para que el sistema diseñado como está no cierto/ ee funcione y funcione bien entonces hay poca dotación de/ de/ de cómo que dijéramos de/ de/ de buses microbuses/ en fin de/ de en el nuevo sistema que/ dificultan tal vez los traslados de las personas/ pero creo que en la esencia ee el hecho de haber/ de haber sectorizado/ ee de haber planificado ee se están optimizando (MIIIH130),

21) me puso un cuchillo en en el pecho/ y me robó una cruz que llevaba de oro encima de/ de la blusa/ y salió corriendo y yo/ bueno obviamente me asusté mucho/ y/ y llegué al local de mi papá llorando y muy muy afligida/ pero gracias a Dios no/ no me pasó nada ni me hirió ni nada pero en fin y esa fue una de las veces porque después me volvieron a asaltar pero ya no fue tan dramático/ o sea fue dramático igual pero sin arma blanca (MIIM123).

\section{En definitiva}

22) hasta que logramos conseguirnos que nos devolvieran la muestra que había sido ee ee analizada digamos por un/ por un experto en estos temas y lo llevamos a otros/ esos otros dijeron de que/ ee no teni/ no estaban seguros digamos de que sí tenía cáncer/ que estaba muy en el límite pero pero pero pero pero que no era maligno/ era benigno/ y después de ese hi/ hizo un un tercer análisis con con cinco expertos y esos cinco expertos def/ en definitiva ee ee dictaminaron digamos que en realidad nunca había tenido cáncer (MAIIIH179),

23) yo creo que un buen amigo es el que/ logra el que logra alegrarse con lo cuan- 
do tú le cuentas algo// que/ que/ te pasa/ qué sé yo/ y cuando también siente empatía cuando tú estás triste/ en definitiva es como cuando le importan/ las cosas que a ti te pasan (MIM121).

\section{En resumen}

24) yo andaba con mis dos hijos/ solo/ y supe que le llamó mucho la atención que yo me preocupara de mis hijos/ las comidas que ee todo lo que se relacionara con ee las vacaciones// perte/ pertenecimos a la misma alianza// en resumen/ llegué de vuelta a Santiago// y ella le redactó la carta al prorrector/ y y me gané al final la beca del cincuenta por ciento (MAIIIH180).

\section{Cuento corto}

25) llegué así/ y puta/ enojado/ ¿cachái?/ porque con mi señora/ puta/ la camioneta al lado ¿cachái?/ acelerando/ le dije "ten cuidado po/ conchetumadre" ¡oh! ¿cachái?/ y llegué y lo pasél después al tiro me pilló como dos semáforos más allál se me ganó al lado y me dijo "¡ah!/ ¿te creí muy chorito?/ ¡qué soi prepotente"/ yo le dije "bueno/ ¿y qué conchetumadre?/ ¿qué? ¿te vai a parar?”/ cuento corto/ apagué la moto/ le puse la pata/ me bajé/ le puse una patada en la puerta/ me subí a la moto y apreté cueva (MBIH051).

Como se aprecia en los ejemplos 1 al 25, los marcadores recapitulativos introducen una conclusión sumaria que compendia lo formulado previamente, facilitando la comprensión del interlocutor. Aunque no constituyen el tipo de reformuladores de mayor frecuencia en la muestra analizada, los recapitulativos presentan la mayor variedad de marcadores, ya que lo conforman 15 unidades ${ }^{10}$. Algunos de estos marcadores muestran evidentes similitudes morfológicas que determinan la existencia de familias léxicas. En este sentido, destaca el amplio grupo de recapitulativos construidos con base en el lexema fin, ya sea como frases preposicionales encabezadas por $a$ o por en. Por una parte, al final y sus variantes a la final y a las finales; a fin de cuentas y su variante al final de cuentas, así como al fin y al cabo y sus variantes al final y al cabo, al final al cabo y a la final y al cabo; por otra, en fin y en definitiva. Parece evidente que, en el primer subgrupo, al final de cuentas ha motivado la variación formal de los otros marcadores, entre los que destaca -por su altísima frecuencia- la variante apocopada al final ${ }^{11}$.

${ }^{10}$ En otro lugar, esperamos presentar los resultados generales del estudio de los marcadores de reformulación incluido en nuestra tesis doctoral; por ahora, baste con señalar que los recapitulativos ocupan el tercer lugar en frecuencia de los reformuladores relevados, después de los explicativos y los de distanciamiento, precediendo solo a los rectificativos.

${ }^{11}$ Por supuesto, casos como los que se ilustran en el siguiente ejemplo, en los que al final no 
A pesar de la bajísima frecuencia de algunos de estos marcadores, en este estudio se ha optado por presentarlos con exhaustividad, con el propósito de ilustrar su notable variedad morfológica. Esta última, a nuestro juicio, da cuenta de que algunos de los recapitulativos del corpus no están completamente gramaticalizados o que se encuentran en vías de estarlo. De hecho, solo los marcadores al fin y al cabo, a fin de cuentas, en resumen y total han sido reconocidos como recapitulativos convencionales en la bibliografía consultada sobre reformuladores del español. En Rojas et al. (2012) ya se había destacado la preponderancia de al final y sus variantes como marcadores de recapitulación, así como la polifuncionalidad de $o$ sea, que también asume esta función. Se agrega aquí cuento corto al paradigma de este tipo de reformuladores que, hasta donde se sabe, parece ser una partícula idiosincrásica del español de Chile.

\subsection{Análisis sociolingüístico de los reformuladores de recapitulación relevados en el corpus}

En esta sección se exponen los resultados del estudio realizado con respecto a la relación de la frecuencia de empleo de los marcadores de reformulación recapitulativa con las tres variables extralingüísticas consideradas en el análisis: sexo, edad y grupo socioeconómico de los sujetos de la muestra. Como ya se señaló, las pruebas de estadística inferencial se aplicarán a los marcadores con 25 o más casos, es decir, al final y o sea con valor recapitulativo.

\subsubsection{Sexo}

En la Tabla II se presentan las frecuencias absoluta y porcentual de la ocurrencia de los marcadores recapitulativos relevados en el corpus, de acuerdo con el sexo de los sujetos.

cumple una función como marcador del discurso, sino que más bien manifiesta un valor gramatical como circunstancial de lugar, no fueron considerados en nuestro análisis:

y el loco me agarró el abrigo/ y me zamarreaba y me decía "entrega el celular/ entrega el celular”/ de ahí mi ex pololo lo empuja del brazo/ ¿cachái?/ y le dice “¡no!/ ¿qué te pasa?”/ y nos corrimos al final/ terminamos al final de la calle/ era una calle que no pasaban autos (MIM105). 
Tabla II. Frecuencia absoluta y porcentaje de los marcadores de reformulación recapitulativa relevados en el corpus según el sexo de los sujetos de la muestra.

\begin{tabular}{|lcc|}
\hline Marcadores & Hombres & Mujeres \\
\hline al final & $96(44,7)$ & $88(44,7)$ \\
\hline a la final & $3(1,4)$ & $0(0)$ \\
\hline a las finales & $9(4,2)$ & $6(3)$ \\
\hline a fin de cuentas & $1(0,5)$ & $0(0)$ \\
\hline al final de cuentas & $0(0)$ & $2(1)$ \\
\hline al fin y al cabo & $1(0,5)$ & $0(0)$ \\
\hline al final y al cabo & $6(2,8)$ & $0(0)$ \\
\hline al final al cabo & $6(2,8)$ & $0(0)$ \\
\hline a la final y al cabo & $1(0,5)$ & $0(0)$ \\
\hline o sea & $63(29,3)$ & $71(36)$ \\
\hline total & $8(3,7)$ & $11(5,6)$ \\
\hline en fin & $8(3,7)$ & $12(6,1)$ \\
\hline en definitiva & $10(4,7)$ & $7(3,6)$ \\
\hline en resumen & $1(0,5)$ & $0(0)$ \\
\hline cuento corto & $2(0,7)$ & $0(0)$ \\
\hline Totales & $215(100)$ & $197(100)$ \\
\hline
\end{tabular}

El análisis de las diferencias entre las medias correspondientes al marcador de recapitulación al final, según el sexo de los sujetos, muestra que la media para los hombres es 1,600, mientras que la de las mujeres alcanza a 1,467. Estos resultados no son significativos estadísticamente, puesto que $\mathrm{F}=0,111, \mathrm{p}=0,740$. La aplicación de la prueba no paramétrica, asimismo, arroja como rangos promedio para los hombres el de 59,64 y para las mujeres el de 61,36. Este mismo análisis confirma la falta de significatividad estadística de estas diferencias (Chi-cuadrado= $0,079, p=0,778)$. El análisis del contraste de las medias correspondientes a la ocurrencia de $o$ sea como marcador de recapitulación, según sexo, por su parte, señala que la media para los hombres es 1,050, mientras que la de las mujeres, 1,183. Sin embargo, los resultados no son significativos estadísticamente $(\mathrm{F}=0,148, \mathrm{p}=$ 0,701). La falta de significatividad estadística de estas diferencias es confirmada por la prueba no paramétrica (Chi-cuadrado $=0,481, \mathrm{p}=0,488)$. Su aplicación, asimismo, da como rangos promedio para los hombres el de 58,52 y para las mujeres el de 62,48. 


\subsubsection{Edad}

La Tabla III expone las frecuencias absoluta y porcentual de la ocurrencia de los marcadores recapitulativos relevados en el corpus, de acuerdo con la edad de los sujetos.

Tabla III. Frecuencia absoluta y porcentaje de los marcadores de reformulación recapitulativa relevados en el corpus según la edad de los sujetos de la muestra.

\begin{tabular}{|lccc|}
\hline Marcadores & 20 a 34 & 35 a 54 & 55 y más \\
\hline al final & $72(50,7)$ & $59(47,2)$ & $53(36,6)$ \\
\hline a la final & $0(0)$ & $3(2,4)$ & $0(0)$ \\
\hline a las finales & $7(4,9)$ & $7(5,6)$ & $1(0,7)$ \\
\hline a fin de cuentas & $1(0,7)$ & $0(0)$ & $0(0)$ \\
\hline al final de cuentas & $0(0)$ & $0(0)$ & $2(1,4)$ \\
\hline al fin y al cabo & $1(0,7)$ & $0(0)$ & $0(0)$ \\
\hline al final y al cabo & $6(4,2)$ & $0(0)$ & $0(0)$ \\
\hline al final al cabo & $6(4,2)$ & $0(0)$ & $0(0)$ \\
\hline a la final y al cabo & $1(0,7)$ & $0(0)$ & $0(0)$ \\
\hline o sea & $39(27,5)$ & $41(32,8)$ & $54(37,2)$ \\
\hline total & $5(3,5)$ & $0(0)$ & $14(9,7)$ \\
\hline en fin & $2(1,5)$ & $3(2,4)$ & $15(10,3)$ \\
\hline en definitiva & $1(0,7)$ & $11(8,8)$ & $5(3,4)$ \\
\hline en resumen & $0(0)$ & $0(0)$ & $1(0,7)$ \\
\hline cuento corto & $1(0,7)$ & $1(0,8)$ & $0(0)$ \\
\hline Totales & $142(100)$ & $125(100)$ & $145(100)$ \\
\hline
\end{tabular}

El análisis de varianza correspondiente al recapitulativo al final, según la edad de los sujetos muestra que la media para el primer grupo (20-34) es 1,800, mientras que la del segundo (35-54) alcanza a 1,475 y la del tercero (55 y más) a 1,325. No obstante, los resultados no son significativos, ya que $F=0,488, p=0,615$. La prueba de Kruskal-Wallis, a su vez, entrega los siguientes rangos promedio de empleo de al final y la edad de los sujetos: $20-34(68,21), 35-54(60,01)$ y 55 y más $(53,28)$, confirmando, además, que se trata de diferencias no significativas (Chi-cuadrado $=4,007, p=0,135)$. En relación con la ocurrencia del marcador recapitulativo $o$ sea y las diferencias etarias de la muestra, la prueba ANOVA indica 
que la media del primer grupo (20-34) es de 0,975, para la del segundo (35-54) de 1,025 y para la del tercero ( 55 y más) de 1,350 . Sin embargo, debido a que $\mathrm{F}=$ $0,459, \mathrm{p}=0,633$, estas diferencias carecen de significatividad. La aplicación de la prueba no paramétrica, asimismo, arroja como rangos promedio para los grupos etarios de la muestra: $20-34(57,45), 35-54(60,71)$ y 55 y más $(63,34)$. Este mismo análisis confirma la falta de significatividad estadística de estas diferencias (Chi-cuadrado $=0,709, \mathrm{p}=0,701)$.

\subsubsection{Grupo socioeconómico}

En la Tabla IV se muestran las frecuencias absoluta y porcentual de la ocurrencia de los marcadores recapitulativos relevados en el corpus, de acuerdo con el grupo socioeconómico de los sujetos.

Tabla IV. Frecuencia absoluta y porcentaje de los marcadores de reformulación recapitulativa relevados en el corpus según el grupo socioeconómico de los sujetos de la muestra.

\begin{tabular}{|llccc|}
\hline Marcadores & B & MB & M & MA \\
\hline al final & $36(42,9)$ & $64(60,1)$ & $25(38,5)$ & $59(37,3)$ \\
\hline a la final & $0(0)$ & $3(2,9)$ & $0(0)$ & $0(0)$ \\
\hline a las finales & $7(8,3)$ & $8(7,6)$ & $0(0)$ & $0(0)$ \\
\hline a fin de cuentas & $0(0)$ & $0(0)$ & $0(0)$ & $1(0,6)$ \\
\hline al final de cuentas & $0(0)$ & $0(0)$ & $0(0)$ & $2(1,3)$ \\
\hline al fin y al cabo & $1(1,2)$ & $0(0)$ & $0(0)$ & $0(0)$ \\
\hline al final y al cabo & $6(7,1)$ & $0(0)$ & $0(0)$ & $0(0)$ \\
\hline al final al cabo & $6(7,1)$ & $0(0)$ & $0(0)$ & $0(0)$ \\
\hline a la final y al cabo & $1(1,2)$ & $0(0)$ & $0(0)$ & $0(0)$ \\
\hline o sea & $11(13,1)$ & $25(23,8)$ & $19(29,2)$ & $79(50)$ \\
\hline total & $14(16,7)$ & $0(0)$ & $3(4,6)$ & $2(1,3)$ \\
\hline en fin & $1(1,2)$ & $3(2,9)$ & $7(10,8)$ & $9(5,7)$ \\
\hline en definitiva & $1(1,2)$ & $0(0)$ & $11(16,9)$ & $5(3,2)$ \\
\hline en resumen & $0(0)$ & $0(0)$ & $0(0)$ & $1(0,6)$ \\
\hline cuento corto & $0(0)$ & $2(2,7)$ & $0(0)$ & $0(0)$ \\
\hline Totales & $84(100)$ & $105(100)$ & $65(100)$ & $158(100)$ \\
\hline
\end{tabular}

El análisis de varianza (ANOVA) de las diferencias entre medias de los grupos socioeconómicos respecto del recapitulativo al final arroja los siguientes datos: 
bajo $(1,200)$, medio bajo $(2,133)$, medio $(0,833)$ y medio alto $(1,967)$, los que no son significativos estadísticamente $(\mathrm{F}=2,489, \mathrm{p}=0,064)$. La prueba de KruskalWallis indica los siguientes rangos promedio: bajo $(51,67)$, medio bajo $(67,30)$, medio $(50,85)$ y medio alto $(72,18)$. Asimismo, señala que estas diferencias son significativas, ya que Chi-cuadrado $=9,502, p=0,023$. Debido a esta discrepancia entre la pruebas paramétrica y no paramétrica, se concluye que se trata de una tendencia que posiblemente se confirme de ampliar el tamaño de la muestra bajo análisis. Para el marcador $o$ sea como reformulador recapitulativo (Gráfico 2) ${ }^{12}$, por su parte, el análisis de varianza de las diferencias entre medias de los grupos socioeconómicos proporciona los siguientes resultados: bajo $(0,367)$, medio bajo $(0,833)$, medio $(0,633)$ y medio alto $(2,633)$, los que son significativos $(\mathrm{F}=$ $11,130, \mathrm{p}=0,000)$.

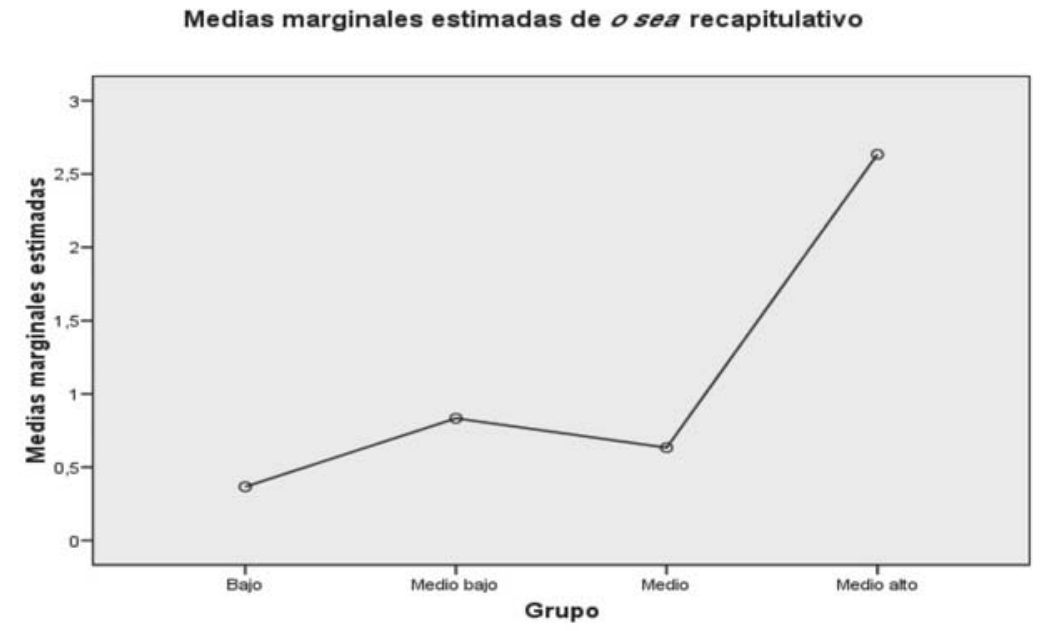

Gráfico 2. Medias marginales estimadas de o sea recapitulativo según grupo socioeconómico.

La significatividad estadística de estas diferencias es confirmada por la prueba no paramétrica (Chi-cuadrado $=28,187, \mathrm{p}=0,000)$. Su aplicación, asimismo, da como rangos promedio para los grupos socioeconómicos: bajo $(44,42)$, medio bajo $(56,55)$, medio $(55,57)$ y medio alto $(85,47)$.

Se puede concluir, según los datos del análisis realizado, que solo las diferencias

${ }^{12}$ Por motivos de espacio, solo se presentará el gráfico del contraste de medias que resultó ser significativo en el análisis estadístico. 
entre los grupos socioeconómicos de los sujetos en el empleo del reformulador recapitulativo $o$ sea resultaron ser significativas estadísticamente, determinando que su uso es preponderante entre los sujetos del grupo medio alto de la comunidad estudiada. No obstante, el empleo mayoritario de al final, por parte de los informantes del mismo grupo socioeconómico, muestra una clara tendencia que, con probabilidad, sería comprobada de ampliar la cantidad de los materiales analizados.

\section{CONCLUSIONES}

A partir de los resultados obtenidos sobre el funcionamiento pragmático-discursivo y la distribución social de los marcadores de reformulación recapitulativa, en el habla capitalina, se pueden sintetizar las principales conclusiones de este estudio. En primer lugar, en los materiales recopilados se identificaron 412 ocurrencias de la función discursiva (variable) "reformulación de recapitulación mediante marcadores", la que se materializó por medio del siguiente paradigma de reformuladores (variantes), con las frecuencias absolutas y porcentuales que se indican: al final $(184=44,7 \%)$, a la final $(3=0,8 \%)$, a las finales $(15=3,6 \%)$, a fin de cuentas $(1=$ $0,2 \%)$, al final de cuentas $(2=0,5 \%)$, al fin y al cabo $(1=0,2 \%)$, al final $y$ al cabo $(6=1,5 \%)$, al final al cabo $(6=1,5 \%)$, a la final y al cabo $(1=0,2 \%)$, o sea $(134=$ $32,5 \%)$, total $(19=4,6 \%)$, en fin $(20=4,9 \%)$, en definitiva $(17=4,1 \%)$, en resumen $(1=0,2 \%)$ y cuento corto $(2=0,5 \%)$. De acuerdo con estos datos, se pudo corroborar la primera hipótesis, esto es, la preponderancia de al final como marcador recapitulativo, aunque el valor recapitulativo de $o$ sea también es muy frecuente. En segundo término, el factor sociodemográfico más sensible al empleo de los reformuladores de recapitulación fue el grupo socioeconómico de los informantes. No se relevaron diferencias de sexo ni de edad significativas, por lo que los mencionados factores resultaron ser irrelevantes, desde el punto de vista estadístico. Estos resultados permitieron comprobar la segunda hipótesis de este estudio, a saber, la irregularidad del influjo de los factores sociodemográficos en el empleo de los reformuladores de recapitulación. De este modo, el empleo de $o$ sea como reformulador recapitulativo está más extendido entre los hablantes del grupo socioeconómico medio alto. Estas diferencias en el uso del mencionado marcador resultaron ser significativas, en términos estadísticos. Por último, se estima que resultaría interesante llevar a cabo trabajos contrastivos con muestras de otras comunidades de habla española que replicaran lo desarrollado en el presente estudio, de manera de comparar el comportamiento de los marcadores de recapitulación -en particular, el de al final y o sea con valor recapitulativo- en otras variedades del español. Así, se contribuiría al conocimiento de la variación socio-dialectal del empleo de los marcadores del discurso en la lengua española, en general. 
Variantes y equivalentes funcionales de al final: los reformuladores de recapitulación en el habla santiaguina / A. SAN MARTíN

\section{REFERENCIAS}

ADIMARK (Investigaciones de Mercado y de Opinión Pública). (2003). Mapa socioeconómico de Chile. Nivel socioeconómico de los hogares del país basado en datos del Censo. Disponible en http://www.adimark.cl/medios/estudios/ mapa_socioeconomico_de_chile.pdf

AIM (Asociación de Investigadores de Mercado). (2008). Grupos socioeconómicos 2008. Disponible en http://www.anda.cl/estudios/textos/DescripcionGSEChile2008.pdf

Anscombre, Jean y Oswald Ducrot. (1994). La Argumentación en la lengua. Madrid, España: Gredos.

Blas Arroyo, José Luis. (2005). Sociolingüistica del español. Desarrollos y perspectivas en el estudio de la lengua española en contexto social. Madrid, España: Cátedra.

Bright, William. (1998). Social factors in language change, en Florian Coulmas (Ed.), The Handbook of Sociolinguistics. Oxford, Reino Unido: Blackweel Publishing, 81-91.

Carbonero, Pedro y Juana Santana. (2010). Marcadores del discurso, variación dialectal y variación social, en Óscar Loureda y Estrella Acín (Coords.), Los estudios sobre marcadores del discurso, hoy. Madrid, España: Arco Libros, 497521.

Cortés, Luis. (1998). Marcadores del discurso y análisis cuantitativo. En María Martín Zorraquino y Estrella Durán (Coords.), Los marcadores del discurso. Teoría y análisis. Pp. 143-160. Madrid, España: Arco Libros.

Garcés Gómez, María Pilar. (2003). Los marcadores de recapitulación y de reconsideración en el discurso. Revista de Investigación Lingüistica, 1 (6), 111-141. . (2008). La organización del discurso: marcadores de ordenación y reformulación. Madrid, España/Frankfurt, Alemania: Iberoamericana/Vervuert. . (2011). Procesos de reformulación en el discurso oral: corrección y rectificación. Español Actual, 96, 89-106.

Gülich, Elizabeth y Thomas Kotschi. (1983). Les marqueurs de reformulation paraphrastique. Cahiers de Linguistique Française, 5, 305-351. . (1995). Discourse Production in Oral Communication. A Study Based on French. En U. Quasthoff (Ed.), Aspects of Oral Communication (pp. 30-66). Berlín, Alemania: De Gruyter.

Hernández Campoy, Juan Manuel y Manuel Almeida. (2005). Metodología de la investigación sociolingüistica. Málaga, España: Editorial Comares.

ICCOM (Instituto Consultor en Comercialización y Mercado). (2005). Descripción Básica de los Niveles Sociales Hogares Urbanos Región Metropolitana. Disponible en http://www.cadem.cl/v1/files/estadistico/descripcionBasica_ GSE_ICCOM_2005.pdf 
Labov, William. (1983). Modelos sociolingüísticos. Madrid, España: Cátedra.

Lavandera, Beatriz. (1978). Where does the sociolinguistic variable stop? Language in Society, 7, 171-182.

Lenski, Gerhardt. (1954). Status Crystallization: A Non-vertical Dimension of Social Status. American Sociological Review, 19, 405-413.

López Morales, Humberto. (1994). Métodos de investigación lingüistica. Salamanca, España: Ediciones Colegio de España. . (2004). Sociolingüística. Madrid, España: Gredos.

Loureda, Óscar y Estrella Acín (Coords.). (2010). Los estudios sobre marcadores del discurso, hoy. Madrid, España: Arco Libros.

Macaulay, Ronald. (2003). Discourse variation. En J. Chambers, P. Trudgill y N. Schilling-Estes (Eds.), The Handbook of Language Variation and Change. Oxford, Reino Unido: Blackwell, 283-305.

Martín Zorraquino, María. (1998). Los marcadores del discurso desde el punto de vista gramatical, en María Martín Zorraquino y Estrella Montolío (Coords.), Los marcadores del discurso. Teoría y análisis. Madrid, España: Arco Libros, 1953.

Martín Zorraquino, María y Estrella Montolío (Coords.). (1998). Los marcadores del discurso. Teoría y análisis. Madrid, España: Arco Libros.

Martín Zorraquino, María y José Portolés. (1999). Los marcadores del discurso, en Ignacio Bosque y Violeta Demonte (Eds.), Gramática descriptiva de la lengua española. Madrid, España: Espasa Calpe, 4051-4207.

Moreno Fernández, Francisco. (1990). Metodología sociolingüistica. Madrid, España: Editorial Gredos.

. (2009). Principios de sociolingüistica y sociología del lenguaje. Barcelona, España: Ariel.

Portolés, José. (2001). Marcadores del discurso. Barcelona, España: Ariel. . (2004). Pragmática para hispanistas. Madrid, España: Síntesis.

Prieto, Luis. (1995-1996). Análisis sociolingüístico del dequeísmo en el habla de Santiago de Chile. Boletín de Filología, XXXV, 379-452.

Rojas, Cristián, Alejandra Rubio, Abelardo San Martín y Silvana Guerrero. (2012). Análisis pragmático y sociolingüístico del empleo de los marcadores discursivos de reformulación en el habla de Santiago de Chile. Lenguas Modernas, 40, 103-123.

Rossari, Corinne. (1994). Les operations de reformulation. Berna, Suiza: Peter Lang. San Martín, Abelardo. (2004-2005). Igual como marcador discursivo en el habla de Santiago de Chile: función pragmático-discursiva y estratificación social de su empleo. Boletín de Filología, XL, 201-232.

. (2011). Los marcadores interrogativos de control de contacto en el corpus PRESEEA de Santiago de Chile. Boletín de Filología, XLVI (2), 135-166. 
Variantes y equivalentes funcionales de al final: los reformuladores de recapitulación en el habla santiaguina / A. SAN MARTíN

. (2013). Los reformuladores de distanciamiento en el corpus PRESEEA de Santiago de Chile. Boletín de Filología, XLVIII (1), 171-199.

San Martín, Abelardo y Silvana Guerrero (en prensa). Estudio Sociolingüístico del Español de Chile (ESECH): recogida y estratificación del corpus de Santiago. Boletín de Filología L (1).

Serrano, María José. (2011). Sociolingüistica. Barcelona, España: Ediciones del Serbal.

Schiffrin, Deborah. (1987). Discourse Markers. Cambridge, Estados Unidos: Cambridge University Press.

. (2003). Discourse Markers: Language, Meaning and Context, en Deborah Schiffrin, Deborah Tannen y Heidi E. Hamilton, The Handbook of Discourse Analysis. Oxford, Reino Unido: Blackwell, 54-75.

Silva-Corvalán, Carmen. (2001). Sociolingüistica y pragmática del español. Washington, Estados Unidos: Georgetown University Press.

Trudgill, Peter y Juan Manuel Hernández Campoy. (2007). Diccionario de sociolingüistica. Madrid, España: Gredos.

Valencia, Alba. (2014a). Introducción. Cuadernos de la ALFAL, 5, 4-12. . (2014b). Marcadores del discurso en Santiago de Chile. Cuadernos de la ALFAL, 5, 246-276. 